\title{
DAÑO GENOTÓXICO EN TRABAJADORES DE MINERÍA ARTESANAL EXPUESTOS AL MERCURIO
}

\author{
Jaime A. Rosales-Rimache ${ }^{1, a}$, Nancy Elizabeth Malca ${ }^{1, b}$, Jhonatan J. Alarcón ${ }^{1, b}$, Manuel Chávez ${ }^{1, c}$, \\ Marco Antonio Gonzáles ${ }^{2, d}$
}

\begin{abstract}
RESUMEN
Objetivos. Determinar el daño genotóxico en trabajadores de una minería artesanal expuestos a mercurio. Materiales $y$ métodos. Estudio observacional de corte transversal, en el cual se evaluaron trabajadores expuestos a mercurio $(n=83)$, de quienes se colectaron células por hisopado bucal para su posterior tinción, revisión microscópica y recuento de micronúcleos y otras alteraciones nucleares. También se colectó orina de $24 \mathrm{~h}$ para la determinación de mercurio inorgánico. Resultados. El $68,7 \%$ de las personas estudiadas fueron de sexo masculino, la media de edad fue de $43 \pm 12,4$ años (rango: 16-76). El tiempo promedio de exposición ocupacional a mercurio fue de 12,1 $\pm 6,7$ años, y el contacto con mercurio fue de 4,1 $\pm 3,6$ kg por persona por día. El 93\% de los evaluados no utilizaban equipos de protección personal durante la manipulación del mercurio. Los resultados del monitoreo biológico evidenciaron que el $17 \%$ de los evaluados presentaron concentraciones de mercurio en orina mayor a los $2,5 \mu \mathrm{g} / \mathrm{L}$; siendo este valor el límite de detección de la técnica de medición utilizada. Los resultados de la evaluación genotóxica evidenciaron que el 15\% de las personas con exposición laboral a mercurio presentaron micronúcleos en células de epitelio bucal; hallándose otros indicadores de alteración nuclear como los puentes nucleoplásmicos, gemaciones y binucleaciones, que también son considerados como eventos genotóxicos asociados a la exposición por agentes de riesgo físico o químico. Conclusiones. El hallazgo de micronúcleos en células del epitelio bucal reflejan daño genotóxico asociado a la exposición laboral por mercurio utilizado en las actividades de minería artesanal.
\end{abstract}

Palabras clave: Pruebas de micronúcleos; Mercurio; Genotoxicidad; Exposición ocupacional; Minería (fuente: DeCS BIREME).

\section{GENOTOXIC DAMAGE AMONG ARTISANAL AND SMALL-SCALE MINING WORKERS EXPOSED TO MERCURY}

\begin{abstract}
Objectives. To determine the genotoxic damage among artisanal and small-scale mining workers exposed to mercury. Materials and methods. Observational cross-sectional study which evaluated mercury-exposed workers $(n=83)$, whose cells were collected by mouth swab for further staining, microscopic observance, micronuclei count, and other nuclear alterations. 24-hour urine was also collected for the determination of inorganic mercury. Results. $68.7 \%$ of participants were male, the mean age being $43 \pm 12,4$ years (range: 16-76). The average time of occupational exposure to mercury was 12,1 $\pm 6,7$ years, and the contact with mercury was $4,1 \pm 3,6 \mathrm{~kg}$ per person per day. $93 \%$ of participants failed to wear personal protection gear while handling mercury. Results of biological monitoring showed that $17 \%$ of participants had concentrations of mercury in urine higher than $2,5 \mu \mathrm{g} / \mathrm{L}$, this value being the detection limit of the measurement technique used. Results of the genotoxic evaluation evidenced that $15 \%$ of people with labor exposure to mercury presented micronuclei in mouth epithelial cells, and other indicators of nuclear alteration such as nucleoplasmic bridges, gemmation and binucleation were found, which are also considered genotoxic events associated to the exposure of physical or chemical risk agents. Conclusions. The finding of micronuclei in mouth epithelial cells reflects genotoxic damage associated to the labor exposure of mercury used in artisanal and small-scale mining activities.
\end{abstract}

Key words: Micronucleus tests; Mercury; Genotoxicity; Occupational exposure; Mining (source: MeSH NLM).

\section{INTRODUCCIÓN}

La minería artesanal es una actividad económica que involucra a más de 70 mil mineros, de ella dependen 400 mil personas y 40 mil familias peruanas que han encontrado una alternativa para combatir el desempleo, con poca inversión, tecnología sencilla y trabajo intensivo. Para esta labor se aprovechan aquellos yacimientos que para la minería convencional han dejado de ser atractivas. Las mejores oportunidades para su desarrollo se dieron en los yacimientos auríferos de Madre de Dios, Puno y del llamado Sur Medio (Ica,

Centro Nacional de Salud Ocupacional y Protección del Ambiente para la Salud, Instituto Nacional de Salud. Lima, Perú.

Centro Nacional de Salud Pública, Instituto Nacional de Salud. Lima, Perú.

Licenciado en Tecnología Médica, magíster en Salud Ocupacional; ${ }^{\mathrm{b}}$ licenciado en Tecnología Médica; ${ }^{\mathrm{c}}$ licenciado en Química; ${ }^{\mathrm{d}}$ licenciado en Estadística Recibido: 16-05-2013 Aprobado: 07-08-13

Citar como: Rosales-Rimache JA, Malca NE, Alarcón JJ, Chávez M, Gonzáles MA. Daño genotóxico en trabajadores de minería artesanal expuestos al mercurio. Rev Peru Med Exp Salud Publica. 2013;30(4):595-600. 
Ayacucho y Arequipa) del Perú. Sin embargo, esta actividad enfrenta múltiples problemas relacionados con la salud de los trabajadores y la población en general ya que los campamentos espontáneos se han convertido en centros poblados desordenados y sin servicios básicos en donde se pone en riesgo la salud de los trabajadores. A este riesgo se suma el riesgo mediado por la contaminación ambiental producto de esta actividad minera.

El mercurio es un metal en estado líquido (1) que se utiliza en el proceso de separación del oro de la arenilla extraída de los socavones mineros. Durante dicho proceso los trabajadores utilizan los quimbaletes para triturar la arenilla que es mezclada con el mercurio líquido, el cual frecuentemente es manipulado sin elementos de protección personal, ello incrementa la exposición a los vapores de mercurio, que ingresan al organismo principalmente por vía respiratoria y ocasionan efectos neurotóxicos, nefrotóxicos y genotóxicos en tejido epitelial de algunas mucosas como la oral y nasal. Es por ello que el mercurio es considerado como un posible carcinógeno para los seres humanos ${ }^{(2)}$. El índice de exposición biológica a mercurio se determina, según la Organización Mundial de la Salud, cuando se encuentran concentraciones iguales o mayores a $50 \mu \mathrm{g}$ en orina de $24 \mathrm{~h}^{(1)}$.

Si se considera que las células epiteliales presentan un ciclo celular corto (1 a 3 semanas), ello implica que estas van exfoliándose constantemente y, por lo tanto, son muestras idóneas para evaluar el daño genético en poblaciones que presentan una exposición reciente hacia algún compuesto genotóxico, tal como el mercurio ${ }^{(3-5)}$. El daño genotóxico ocasionado por la exposición al mercurio puede ser medido a través del uso de biomarcadores de efecto temprano, tales como la formación de micronúcleos en células epiteliales de la mucosa bucal ${ }^{(6,7)}$.

Los micronúcleos son indicadores de daño cromosómico numérico y estructural. Es decir, permiten evaluar la aneugenicidad y clastogenicidad de una sustancia. Estos daños ocurren durante la etapa de mitosis, por una rotura de cromatídica o por la no migración cromosómica durante la anafase; y se manifiesta como un pequeño núcleo en la célula interfásica. En los últimos años, la determinación de micronúcleos en distintas células como las epiteliales de exfoliación ha cobrado mayor importancia, sobre todo en grupos de riesgo con exposición a agentes genotóxicos y carcinogénicos ${ }^{(8)}$. Ello, debido a que hay evidencia que señala que la presencia de micronúcleos es capaz de evaluar clastogenicidad y aneugenicidad al mismo tiempo ${ }^{(9)}$. Los micronúcleos permiten también valorar la inestabilidad genética, la cual es inducida por la exposición de distintos agentes genotóxicos ${ }^{(10)}$ y su uso ya ha sido validado internacionalmente ${ }^{(11)}$. Además, algunas investigaciones señalan que pueden ser usados incluso como marcadores de riesgo relativo de padecer cáncer en personas con exposición a agentes genotóxicos ${ }^{(12)}$ o como marcadores de riesgo para cáncer cervical ${ }^{(13)}$.

En salud ambiental, la determinación de micronúcleos también permite evaluar personas con exposición a agentes contaminantes como los metales pesados (14). En un estudio previo, un grupo de investigadores encontraron que el recuento de micronúcleos en personas expuestas a mercurio era significativamente superior al grupo control ${ }^{(15)}$. Las referencias sobre la determinación de micronúcleos como marcador de genotoxicidad es tan extensa, que incluso se ha planteado su inclusión como herramienta de monitoreo biológico en los programas de vigilancia epidemiológica molecular en cáncer ${ }^{(16)}$. Sin embargo, hay poca información que indique la asociación entre la exposición laboral a metales pesados, como el mercurio, y la frecuencia de micronúcleos en células de epitelio bucal, aun cuando estas son de fácil acceso y no requieren de procedimientos de cultivo celular para su cuantificación y estudio citogenético ${ }^{(17)}$. Es por ello que el objetivo del presente estudio fue determinar el daño genotóxico, a través de la determinación de micronúcleos en muestras de hisopado de epitelio bucal de trabajadores de una minería artesanal expuestos a mercurio.

\section{MATERIALES Y MÉTODOS}

Se realizó un estudio de tipo observacional de corte transversal. Para ello, durante el mes de mayo de 2010, se evaluaron trabajadores de minería artesanal con exposición laboral a mercurio en la localidad de Tulín (a $460 \mathrm{~km}$ de la ciudad de Lima), perteneciente al distrito del Ingenio en la provincia de Nazca, departamento de Ica, Perú. Para el cual se realizó un muestreo no probabilístico. Se incluyeron a todos aquellos trabajadores que aceptaron participar de manera voluntaria, previa sensibilización sobre la problemática a investigar. Se excluyeron a personas con enfermedades degenerativas, cáncer, o que hayan estado expuestas a rayos $\mathrm{X}$ durante los treinta días previos al estudio.

Para la recolección de datos se aplicó una ficha epidemiológica dirigida a la obtención de datos demográficos, laborales y de bioseguridad asociados a la exposición por mercurio. Para evaluar la genotoxicidad en personas con exposición laboral a mercurio se realizó el recuento de micronúcleos en células de epitelio bucal. Para ello se obtuvieron muestras de hisopado bucal que fueron resuspendidas en suero fisiológico, para luego ser fijadas en líquido de Carnoy y montadas en láminas 
portaobjeto; para su lectura se empleó tinción con Giemsa y recuento microscópico en 1000 células. Asimismo, para la determinación de mercurio se colectó orina durante un periodo de 24 horas. Las muestras fueron preservadas entre $2-8{ }^{\circ} \mathrm{C}$ hasta su análisis en el Laboratorio Clínico y Toxicológico del Centro Nacional de Salud Ocupacional y Protección delAmbiente para la Salud del Instituto Nacional de Salud del Perú. La determinación cuantitativa de los niveles de mercurio se realizó empleando la metodología de la espectrofotometría de absorción atómica con vapor frío. Se siguió el procedimiento establecido por el Centro de Control y Prevención de Enfermedades de los Estados Unidos (CDC - Método de referencia: 1180B/05-OD). La población fue dividida en dos grupos: sin concentración de mercurio en orina ( $<2,5 \mathrm{ug}$ ), y con concentración de mercurio en orina ( $\geq 2,5 \mathrm{ug}$ ), se fijó este valor como límite dado que este corresponde al límite de detección de la metodología de medición.

El plan de análisis incluyó, además del cálculo de las frecuencias (absolutas y relativas) y de las medidas de tendencia central, estadística bivariada, como las pruebas t de Student y la U de Mann-Whitney. Se realizó también, regresión logística multinomial para evaluar la asociación entre las variables sociodemográficas y otras variables de interés, con la finalidad de modelar la probabilidad del comportamiento de las variables dependientes en función a los niveles de mercurio en orina, asimismo, se realizó un análisis de regresión logística multinomial en donde la variable dependiente fue el recuento de micronúcleos y la variable independiente fue la presencia de mercurio en orina. Se empleó el paquete estadístico SPSS v 19.0.

Todos los participantes recibieron previo al estudio una charla de sensibilización sobre la importancia del estudio, tras lo cual firmaron un consentimiento informado voluntariamente. El informe técnico de la evaluación fue entregado a la dirección del Puesto de Salud en Tulín. Todos los participantes del estudio fueron informados de los procedimientos a realizar, riesgos, beneficios y la confidencialidad de los resultados.

\section{RESULTADOS}

Se incluyeron un total de 83 trabajadores de una minera artesanal, el 68,7\% de ellos fueron varones. La media de edad fue de $43 \pm 12,4$ años (rango: 16-76). El promedio del tiempo de exposición ocupacional a mercurio fue de $12,1 \pm 6,7$ años (rango: 1-32), en tanto que la mediana de exposición ocupacional a mercurio por semana fue de 12,0 horas (rango: 8-60). La media de la cantidad de mercurio empleado en los quimbaletes por cada trabajador fue de 4,1 $\pm 3,6 \mathrm{~kg}$ por día (rango: 2-20).
Tabla 1. Características del tipo de trabajo y condiciones de bioseguridad

\begin{tabular}{|c|c|}
\hline & N. ${ }^{\circ}(\%)$ \\
\hline \multicolumn{2}{|l|}{ Tipo de trabajo } \\
\hline Quimbaletero & $24(28,9)$ \\
\hline Quimbaletero-obrero de interior de minas & $53(63,9)$ \\
\hline Quimbaletero-agricultor & $3(3,6)$ \\
\hline Quimbaletero-almacenero de mercurio & $3(3,6)$ \\
\hline \multicolumn{2}{|l|}{ Uso de la misma vestimenta de trabajo } \\
\hline Sí & $43(51,8)$ \\
\hline No & $40(48,2)$ \\
\hline \multicolumn{2}{|l|}{ Frecuencia de cambio de vestimenta } \\
\hline Diario & $39(47)$ \\
\hline Interdiario & $32(38,6)$ \\
\hline >2 días/semana & $12(14,5)$ \\
\hline \multicolumn{2}{|l|}{ Uso de EPP } \\
\hline Mandil & $1(1,2)$ \\
\hline Guantes & $1(1,2)$ \\
\hline Mascarillas & $2(2,4)$ \\
\hline Gorros & $2(2,4)$ \\
\hline No usa EPP & $77(92,8)$ \\
\hline
\end{tabular}

EPP: equipo personal de protección

El $51,8 \%$ de los evaluados manifestaron usar la vestimenta de trabajo tanto en su centro de labores como en sus hogares. El $14,5 \%$ de los trabajadores tuvieron una frecuencia de cambio de vestimenta mayor a 2 días por semana. Además, el 92,8\% de los evaluados no utilizaban ningún equipo de protección personal, y solo en casos muy aislados utilizaron mandil, guantes, mascarillas y gorros (Tabla 1).

En base al límite de detección de la metodología de medición, se encontró que el 16,9 \% de los evaluados presentaron concentraciones de mercurio en orina mayor a 2,5 $\mu \mathrm{g} / \mathrm{L}$. Al realizar la comparación entre los tiempos promedios laborando y los niveles de mercurio detectados en orina (Figura 1), se encontró que el promedio de tiempo laborando en el grupo en que no se detectó mercurio en orina fue de 11,6 \pm 6,5 años (rango:

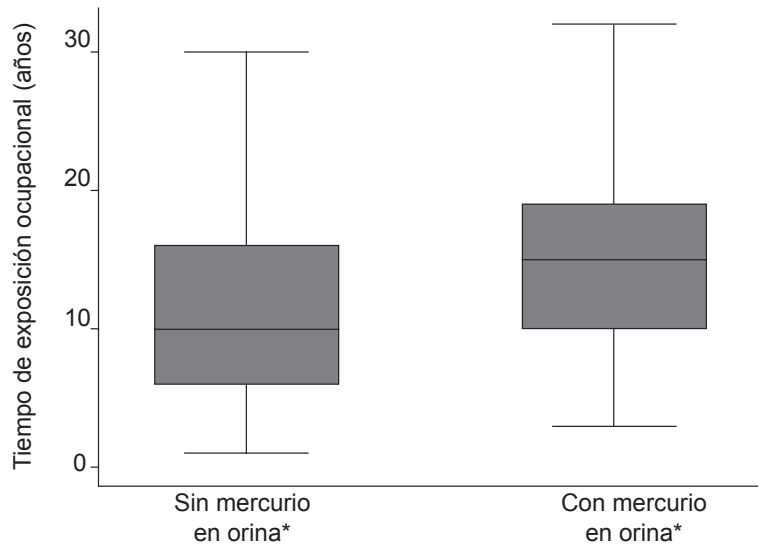

Figura 1. Presencia de mercurio en orina según tiempo de exposición laboral

* El punto de corte está fijado en 2,5 ug/L de mercurio en orina, límite de detección de mercurio por espectrofotometría de absorción atómica con vapor frío. 


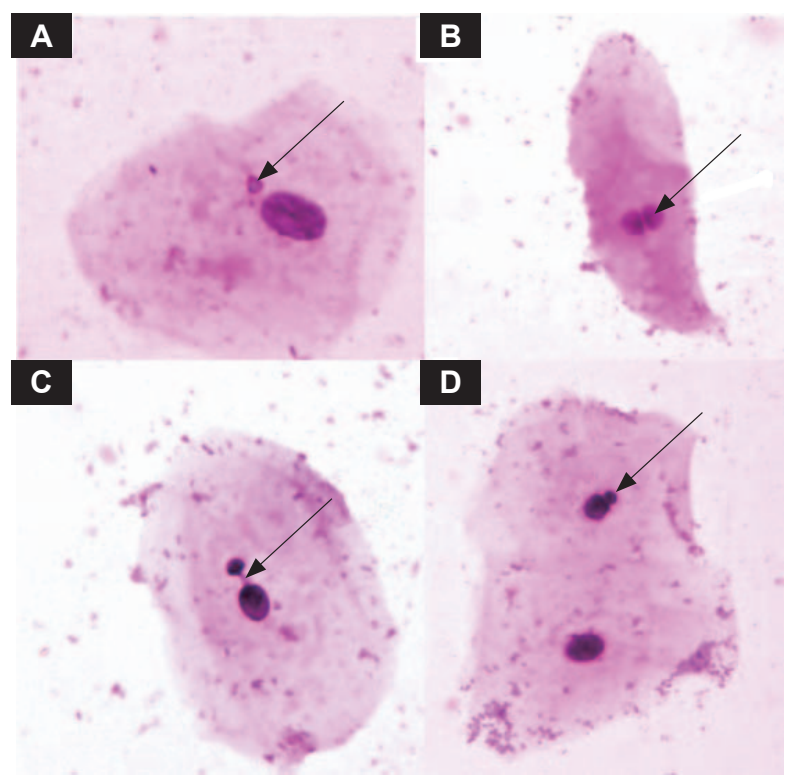

Figura 2. Micronúcleos y alteraciones nucleares en células de epitelio bucal. Las flechas señalan: a) micronúcleo; b) binucleación; c) puentes nucleoplásmicos; d) gemación (Giemsa, 100 X)

1-30) y el tiempo promedio en el grupo en el cual se detectó orina fue de 14,6 \pm 7,1 años (rango: 3-32). Se encontró además que la media de la concentración de mercurio en orina de 24 horas fue de 5,7 ug/L. Asimismo se encontró que para los percentiles 5, 10, 25, 75, 90 y

Tabla 2. Regresión logística multinomial para presencia de mercurio en orina

\begin{tabular}{lccc}
\hline & \multirow{2}{*}{ OR } & \multicolumn{2}{c}{ IC 95\% } \\
\cline { 3 - 4 } & & LS & LI \\
\hline Edad & 0,97 & 0,92 & 1,02 \\
$\quad$ años & & & \\
$\begin{array}{l}\text { Sexo* } \\
\quad \text { Mujer }\end{array}$ & 0,31 & 0,06 & 1,51 \\
$\begin{array}{l}\text { Área de trabajo** } \\
\quad \begin{array}{l}\text { Quimbaletero y obrero de interior } \\
\text { de mina }\end{array}\end{array}$ & 2,56 & 0,52 & 12,71 \\
$\quad \begin{array}{l}\text { Quimbaletero y almacenero } \\
\text { Tiempo de exposición } \\
\text { ocupacional } \\
\quad \text { Años }\end{array}$ & 22,00 & 1,33 & 362,92 \\
$\begin{array}{l}\text { Cantidad Hg /empleado } \\
\quad \text { Kilos }\end{array}$ & 1,07 & 0,98 & 1,17 \\
\hline $\begin{array}{l}\text { Horas de exposición semanal } \\
\quad \text { Horas }\end{array}$ & 1,02 & 0,88 & 1,19 \\
\hline $\begin{array}{l}\text { Misma vestimenta de trabajo } \\
\quad \text { No }\end{array}$ & 0,99 & 0,96 & 1,02 \\
\hline $\begin{array}{l}\text { Frecuencia de cambio de } \\
\text { vestimentatt } \\
\quad \text { Interdiario }\end{array}$ & 0,77 & 0,24 & 2,46 \\
\hline > 2 días/semana & & & \\
\hline
\end{tabular}

Categorías de referencia: * varón, ${ }^{* *}$ quimbaletero, ${ }^{\dagger} \mathrm{si},{ }^{\dagger \dagger}$ diario.

OR: Odds Ratio, LS: límite superior, LI: límite inferior
Tabla 3. Regresión logística multinomial para recuento de micronúcleos y alteraciones nucleares en mineros con presencia de mercurio en orina.

\begin{tabular}{lccc}
\hline & \multirow{2}{*}{ OR } & \multicolumn{2}{c}{ IC 95\% } \\
\cline { 3 - 4 } & & LS & LI \\
\hline Micronúcleos Bucal & & \\
$\quad$ Uno & 22,10 & 4,80 & 101,70 \\
$\quad$ Dos & 12,60 & 1,50 & 109,40 \\
$\begin{array}{l}\text { Puente Nucleoplásmico } \\
\text { Uno }\end{array}$ & 5,40 & 0,70 & 42,30 \\
$\quad$ Dos & - & - & - \\
$\begin{array}{l}\text { Gemación } \\
\quad \text { Uno }\end{array}$ \\
$\begin{array}{l}\text { Dos } \\
\text { Binucleación celular } \\
\quad \text { Uno }\end{array}$ \\
$\begin{array}{l}\text { Dos } \\
\quad \text { Más de dos }\end{array}$ & 3,40 & 0,72 & 16,01 \\
\hline
\end{tabular}

OR: Odds Ratio, LS: límite superior, LI: límite inferior

95, estas concentraciones fueron: 2,6, 2,8, 3,9, 7,4, 8,4 y $8,7 \mathrm{ug} / \mathrm{L}$ respectivamente.

Los resultados de la evaluación genotóxica evidenciaron que el $18,1 \%$ de las personas con exposición laboral a mercurio presentaron micronúcleos en las células de epitelio bucal, de ellos el $73,3 \%$ (11/15) presentaron solo un micronúcleo y el $26,7 \%$ (11/15) presentaron dos micronúcleos. También pudieron ser evidenciadas otras alteraciones nucleares (Figura 2), tales como: puente nucleoplásmico $(7,2 \%)$, gemación $(15,6 \%)$ y binucleación celular $(21,7 \%)$. Los resultados de las regresiones logísticas multinomiales se presentan en las Tablas 2 y 3.

\section{DISCUSIÓN}

Tulín es uno de los centros poblados más representativos de la costa sur del Perú en donde se practica la minería artesanal. Su población es de casi 2000 habitantes, y la minería artesanal es la principal fuente de trabajo debido a la cercanía de algunos centros mineros. Los pobladores de Tulín se dedican a la extracción de oro usando los quimbaletes (molinos artesanales de piedra tallada similar a los batanes) en los cuales amalgaman la arenilla obtenida de los socavones con el mercurio líquido; de este modo extraen el oro en recipientes que luego son llevados a una retorta comunal que fue instalada con el objetivo de minimizar los efectos nocivos de contaminación por emisiones gaseosas de mercurio y posibilitar la recuperación del vapor de mercurio en un 80 a 90\%.

El uso de mercurio en la minería artesanal es muy variable y depende de la cantidad de mineral extraído 
de los socavones. Generalmente, las personas que trabajan dentro de los socavones son las mismas que extraen el oro en los quimbaletes usando el mercurio y, por ende, están expuestos a un mayor riesgo genotóxico manifestado por un incremento en la frecuencia de micronúcleos en células epiteliales bucales. En nuestro estudio se encontró que el uso de los elementos de protección personal en dichos trabajadores es casi nulo, razón por la cual la principal vía de ingreso es la inhalatoria. Ello debido a que el mercurio puede generar vapores a temperatura ambiental y que esta aumenta cuando se realiza los procesos de quema del amalgamado (aleación de mercurio y oro). Otra forma de ingreso del mercurio al organismo es a través de la vía dérmica por manipulación directa del mercurio; aunque esta última en menor proporción (1).

Tal como se ha descrito, los quimbaleteros son los que se encuentran principalmente expuestos a mercurio; en este estudio se observó que ellos, además, pueden ejercer otras tareas que incrementan el tiempo de exposición al mercurio y, por tanto, su riesgo. Tal es así, que aquellos que además trabajan dentro de las minas tienen una probabilidad tres veces mayor de presentar niveles de mercurio en orina, con respecto a quien solo tiene la labor de quimbaletero. En tanto que, los quimbaleteros, que además son almaceneros, tienen una probabilidad de 22 veces más de presentar niveles de mercurio en orina con respecto a uno que solo se dedica a quimbaletero. La amplitud de los intervalos de confianza se debería al tamaño de la muestra entre los quimbaleteros que son también almaceneros. Del mismo modo, se observó que las personas con niveles de mercurio en orina presentan una probabilidad 22 veces mayor de presentar un micronúcleo en la muestra bucal con respecto a los que no presentan niveles de mercurio en orina. Asimismo, se observa que el mismo grupo tiene una probabilidad de 13 veces mayor de presentar dos micronúcleos con respecto a los que no presentan niveles de mercurio en orina. El mismo análisis se aplicó para las alteraciones nucleares (puentes nucleoplásmicos, gemaciones y binucleaciones); sin embargo, en ninguna de estas se encontró evidencia de asociación significativa, probablemente por el tamaño reducido de la muestra.

Por otro lado, es importante señalar que en las personas en quienes se encontraron niveles de mercurio en orina tuvieron una media de mercurio de $5,7 \mathrm{ug} / \mathrm{L}$, valor que es mucho mayor que los valores de referencia indicados para la población general según el Fourth National Report on Human Exposure to Enviromental Chemicals ${ }^{(18)}$ la cual señala que la media de referencia es 3,9 ug/L, pudiéndose observar que en todos los deciles superiores a la media geométrica las mediciones puntuales y por intervalos son superiores a los valores de referencia en la población general de los Estados Unidos en este estudio (Tabla 4).

Los resultados del presente estudio sugieren que existiría asociación entre la presencia de micronúcleos, como marcador de genotoxicidad, y la exposición laboral de los mineros artesanales al mercurio. Este hallazgo es similar a otros estudios realizados, con la diferencia que se asociaron frecuencia de micronúcleos y exposición a agentes genotóxicos ${ }^{(17,19)}$.

Durante el análisis de las muestras biológicas se presentaron algunas limitaciones tales como la poca cantidad de células que se obtuvieron mediante el hisopado bucal lo que dificultó la lectura y toma de fotografías; así mismo, también tuvieron que rechazarse muestras de orina que no cumplieron con los criterios de aceptabilidad como el que sea colectado en un periodo de $24 \mathrm{~h}$. Las muestras de orina puntual no fueron consideradas en el presente estudio.

En conclusión, el hallazgo de micronúcleos en células del epitelio bucal reflejan daño genotóxico asociado a la exposición laboral por mercurio utilizado en las actividades de minería artesanal. Consideramos que la detección de micronúcleos en epitelio bucal es una herramienta costo-eficiente para el monitoreo biológico en los programas de vigilancia epidemiológica molecular en salud ocupacional.

Tabla 4. Niveles de mercurio en orina $(\mu \mathrm{g} / \mathrm{L})$ de los trabajadores evaluados y rangos de referencia internacionales

\begin{tabular}{|c|c|c|c|c|c|c|}
\hline \multirow{3}{*}{ Percentil } & \multicolumn{3}{|c|}{ Encontrado en el estudio } & \multicolumn{3}{|c|}{ Valores de referencia del FNRHEEC* } \\
\hline & \multirow{2}{*}{$\begin{array}{c}\text { Mercurio } \\
\text { (ug/L) }\end{array}$} & \multicolumn{2}{|c|}{ IC95\% } & \multirow{2}{*}{$\begin{array}{c}\text { Mercurio } \\
\text { (ug/L) }\end{array}$} & \multicolumn{2}{|c|}{ IC95\% } \\
\hline & & LS & LI & & LS & LI \\
\hline 5 & 2,65 & 2,65 & 3,84 & - & - & - \\
\hline 10 & 2,81 & 2,65 & 4 & - & - & - \\
\hline 25 & 3,86 & 2,68 & 5,64 & - & - & - \\
\hline 50 & 5,67 & 3,86 & 7,33 & 0,4 & 0,36 & 0,45 \\
\hline 75 & 7,37 & 5,69 & 8,65 & 0,85 & 0,77 & 0,91 \\
\hline 90 & 8,38 & 7,02 & 8,71 & 1,53 & 1,3 & 1,81 \\
\hline 95 & 8,71 & 7,67 & 8,71 & 2,42 & 2,07 & 2,72 \\
\hline
\end{tabular}

\footnotetext{
* Fourth National Report on Human Exposure to Environmental Chemicals
} 
Agradecimientos: a los participantes del estudio, personal de campo y equipo de trabajo que colaboraron activamente en la ejecución del presente estudio de investigación.

Contribuciones de autoría: JAR y NEM han participado en la concepción, diseño y redacción del artículo. JAR, MC y MAG participaron en la revisión y redacción del artículo. JJA y NEM realizaron la recolección de datos, JAR y MAG el análisis de datos y asesoría estadística. Todos los autores aprobaron la versión final a publicar

Fuentes de financiamiento: Instituto Nacional de Salud.

Conflictos de interés: los autores declaran no tener conflictos de interés.

\section{REFERENCIAS BIBLIOGRÁFICAS}

1. U.S. Department of Health and Human Services. Toxicological profile for mercury [Internet]. Atlanta, Georgia: Agency for Toxic Substances and Disease Registry; 1999 [citado 25 de marzo de 2013]. Disponible en: http://www.atsdr.cdc.gov/ToxProfiles/ tp46.pdf

2. World Health Organization, International Agency for Research on Cancer. IARC Monographs on the Evaluation of Carcinogenic Risks to Humans: Beryllium, Cadmium, Mercury, and Exposures in the Glass Manufacturing Industry [Internet]. Geneva: WHO; 1993 [citado 17 de abril de 2013]. Disponible en: http://monographs.iarc.fr/ENG/ Monographs/vol58/volume58.pdf

3. Tolbert PE, Shy CM, Allen JW. Micronuclei and other nuclear anomalies in buccal smears: a reid test in snuff users. Am J Epidemiol. 1991;134(8):840-50.

4. Zabinski Z, Panek A, Wierzewska A, Kasper E, Dyga W, Moszczynski P, et al. Influence of occupational exposure to mercury vapours on lymphocytes susceptibility to the induction of genetic damage. Kraków, Poland: Institute of Nuclear Physics; 2001.

5. Cebulska-Wasilewska A, Panek A, Zabiński Z, Moszczyński P, Au WW. Occupational exposure to mercury vapour on genotoxicity and DNA repair. Mutat Res. 2005;586(2):10214.

6. Albiano NF. Toxicología laboral: criterios para la vigilancia de los trabajadores expuestos a sustancias químicas peligrosas [Internet]. Organización Iberoamericana de Seguridad Social; 2000 [citado 24 de abril de 2013]. Disponible en: http:// www.oiss.org/estrategia/IMG/pdf/ productos_quimicos.pdf

7. Clarkson TW, Magos L, Myers GJ. The toxicology of mercury-current exposures and clinical manifestations. N Engl J Med. 2003 Oct 30;349(18):1731-7.

8. Suhas S, Ganapathy KS, Gayatri Devi M, Ramesh C. Application of the micronucleus test to exfoliated epithelial cells from the oral cavity of beedi smokers, a high-risk group for oral cancer. Mutat Res. 2004 Jul 11;561(1-2):15-21.

9. Norppa H, Luomahaara $S$, Heikanen H, Roth S, Sorsa M, Renzi L, et al. Micronucleus assay in lymphocytes as a tool to biomonitor human exposure to aneuploidogens and clastogens. Environ Health Perspect. 1993;101 Suppl 3:139-43..

10. Zalacain M, Sierrasesúmaga L, Patiño A. El ensayo de micronúcleos como medida de inestabilidad genética inducida por agentes genotóxicos. An Sist Sanit Navar. 2005;28(2):227-36.

11. Fenech M, Holland N, Chang WP, Zeiger E, Bonassi S. The Human MicroNucleus Project--An international collaborative study on the use of the micronucleus technique for measuring DNA damage in humans. Mutat Res. 1999;428(1-2):271-83.

12. Fenech M. Biomarkers of genetic damage for cancer epidemiology. Toxicology. 2002 Dec 27;181182:411-6.

13. Gandhi P, Sharma P, Kaur and Badaruddoza A. The micronucleus test in urothelial cells and uterine smears of cervix cancer patients: a comparison. Int J Hum Genet. 2003;3(2):121-6.
14. Bérces J, Otos M, Szirmai S, CraneUruena C, Köteles GJ. Using the micronucleus assay to detect genotoxic effects of metals ions. Environ Health Perspect. 1993;101 Suppl 3:11-3.

15. Shamy MY, El-Gazzar RM, Taleb AN, Christie NT, El Said KF. Somatic cell mutation in workers occupationally exposed to mercury vapors. J Environ Pathol Toxicol Oncol. 1995;14(34):165-71.

16. Collins AR. Molecular epidemiology in cancer research. Mol Aspects Med. 1998;19(6):359-432.

17. Letaj K, Elezaj I, Selimi Q, Kurteshi K. The effects of environmental pollution with heavy metals in frequency of micronuclei in epithelial buccal cells of human population in Mitrovica. Journal of Chemical Health Risks. 2012;2(3):1-4

18. Centers for Disease Control and Prevention. Fourth National Report on Human Exposure to Environmental Chemicals [Internet]. CDC; 2009 [citado el 27 de abril de 2013]. Disponible en http://www.cdc.gov/ exposurereport/pdf/FourthReport.pdf

19. Ray MR, Basu C, Mukherjee S, Roychowdhury S, Lahiri T. Micronucleus frequencies and nuclear anomalies in exfoliated buccal epithelial cells of firefighters. Int J Hum Genet. 2005;5(1):45-8.

Correspondencia: Jaime Alonso Rosales

Rimache

Dirección: Calle Las Amapolas 350, Lima

14, Perú.

Teléfono: 7480000 anexo 7706, 7717 y 7725

Correoelectrónico:jrosales@ins.gob.pe 